\title{
Index Method of Assessment of the Acoustical Climate in Industrial Rooms
}

\author{
Zbigniew Engel ${ }^{\dagger}$ and Janusz Piechowicz \\ Department of Mechanics and Vibroacoustics, University of Mining and Metallurgy, \\ Al. Mickiewicza 30, 30-059 KrakCw, Poland
}

(Received 15 August 2000; accepted 29 September 2000)

\begin{abstract}
Noise is one of the main problems inside industrial rooms. Therefore minimising the noise levels is essential for the creation of the proper acoustical climate for working conditions. The problem of the assessment of the acoustical conditions in industrial rooms has considerable practical significance. One solution of the problem is suitable analysis of the model of the acoustical field inside industrial rooms. Such a model needs to take into account room shape, engineering processes, work place locations and layout and the interrelation of various noise sources. Index method of the assessment of the acoustics of industrial rooms is connected with the modernisation of the ways of the identification and estimation of occupational risks caused by excessive noise. It is the activity directed towards the protection of work environment and the possibility of improvement of work conditions.
\end{abstract}

${ }^{\dagger}$ Member of the International Institute of Acoustics and Vibration (IIAV)

\section{INTRODUCTION}

The generation and propagation of sound waves inside industrial rooms are very complicated phenomena. We are dealing here with the most complicated types of the spatial vibro-acoustic systems in which the propagation of sound depends on several factors such as: the vibro-acoustic processes with occur in machines and the shape texture and acoustical properties of surrounding surfaces. The main difficulty in analysing such systems is the lack of adequate theoretical models which can take into account the simultaneous influence of all the parameters mentioned.

The design requirements concerning the acoustical safety of work places force continuous improvements in the way the results of model and experimental investigations are presented. It is also the reason for looking for a method of the synthetic evaluation acoustical quality of an industrial room which will allow one to assess the occupational risk for noise-exposed persons.

The criterion for the correctness of the noise assessment indices provides a good correlation between the degree of hazard and the numerical value of the index. However the final verification of the interior of an industrial room must be made during its use. Certain assessments must be performed at each stage of the design process and especially at the end of the preliminary design. One of the most significant parameters for a proper arrangement of an industrial room interior is its acoustical climate.

The acoustical climate of a work environment describes all of the acoustical phenomena which occur in that environment. The climate is usually estimated from several indices which are functions of frequency, time and space.

The acoustical climate, $K_{A}$ is a function of partial climates and can be expressed as:

$$
K_{A}=F\left(K_{a_{1}}, K_{a_{2}}, \ldots, K_{a_{n}}\right),
$$

where $K_{a_{1}}, K_{a_{2}}, \ldots, K_{a_{n}}$ are the partial climates present in a particular zone of an environment.
Each partial climate depends on the noise level generated by separate sources belonging to a specified group of sound sources:

$$
K_{a_{i}}=f\left(L_{H_{i}}, \Delta_{L T}\right) \quad i=1,2, \ldots, n
$$

where $L_{H_{i}}$ is the sound pressure level (or sound power level) of separate noise sources of the specified group at a certain observation point; $\Delta_{L T}$ is the total reduction of the sound pressure level (or sound power level) caused by all of the parameters of the work environment.

The quality of the acoustical climate in a work environment is defined by an equivalent sound level $L_{A_{e q} T}$ which is characterised by a high correlation with noise annoyance. The equivalent sound level enables the estimation of noises of different natures and also provides the possibility to find functions relating values of $L_{e q}$ with parameters describing the work environment.

Noise assessment indices allow one to estimate hazard results on the basis of known physical features of the noise. Such features can be not only measured but also predicted or simulated. They give the possibility of predicting the acoustical climate of newly designed rooms and of choosing the best solutions for their acoustical performance. The acoustical climate index can be an essential parameter for the estimation of the occupational risk for noise-exposed employees. The identification of the noise hazard (noise measurements and their comparison with permissible noise values) and the recognition of the possibility of that hazard reduction are required for the estimation of the noise hazard. That estimation provides also information on where and what means should be applied to improve a local or general acoustical climate in the room so as to reduce the occupational risk to a reasonable and consciously acceptable level.

Criteria for the acoustical climate assessment in industrial rooms should be simple. This means they should contain as small as possible a number of parameters that result in the estimation (e.g. single-numbered index) and a large number of 